\title{
3-529-4
}

\section{Molecular mechanism underlying myonuclei accretion during resistance training}

\section{So-Ichiro Fukada}

\section{Graduate School of Pharmaceutical Sciences, Osaka University}

Skeletal muscle mainly consists of multinuclear cells named myofibers. It is well established that skeletal muscle is essential for energy metabolism, physical activity, and sedentary activity (breathing and swallowing). The physical activity has great impacts on properties of myofiber, and the myofiber-derived factor (called myokines or exerkine) has systemic effects across species.

Vigorous physical activity like resistance training induces myofiber hypertrophy. Importantly, resistance training induces the increased number of myonuclei, which is responsible for efficient myofiber hypertrophy and the effect of repeated-training. Skeletal muscle-specific stem cells, muscle satellite cells (MuSCs), is the only cell that can physiologically supply new myonuclei.

In a steady condition, MuSCs are maintained in a dormant state, but when skeletal muscle perceive mechanical load, MuSCs escape from the dormant state and start to proliferate. By response to the resistance training, MuSCs eventually supply new myonuclei. The regulation of MuSCs behaviors is critical for efficient muscle hypertrophy, but the molecular mechanisms regulating the MuSC behaviors remain to be elucidated. In this symposium, I would like to present our data suggesting the missing link between physical activity and MuSC behaviors. 